\title{
BMJ Open PARROT Ireland: Placental growth factor in Assessment of women with suspected pre-eclampsia to reduce maternal morbidity: a Stepped Wedge Cluster Randomised Control Trial Research Study Protocol
}

Deirdre Hayes-Ryan, ${ }^{1,2}$ Karla Hemming, ${ }^{3}$ Fionnaula Breathnach, ${ }^{4}$ Amanda Cotter, ${ }^{5}$ Declan Devane,${ }^{6,7}$ Alyson Hunter, ${ }^{8}$ Fionnuala M McAuliffe, ${ }^{9}$ John J Morrison, ${ }^{10}$ Deirdre J Murphy, ${ }^{11}$ Ali Khashan, ${ }^{12,13}$ Brendan McElroy, ${ }^{14}$ Aileen Murphy, ${ }^{14}$ Eugene Dempsey, ${ }^{13,15}$ Keelin O'Donoghue, ${ }^{2,13}$ Louise C Kenny ${ }^{13,16}$

To cite: Hayes-Ryan $D$, Hemming K, Breathnach F, et al. PARROT Ireland: Placental growth factor in Assessment of women with suspected pre-eclampsia to reduce maternal morbidity: a Stepped Wedge Cluster Randomised Control Trial Research Study Protocol. BMJ Open 2019;9:e023562. doi:10.1136/ bmjopen-2018-023562

- Prepublication history for this paper is available online To view these files, please visit the journal online (http://dx.doi. org/10.1136/bmjopen-2018023562).

Received 12 April 2018 Revised 2 October 2018 Accepted 17 December 2018

Check for updates

(C) Author(s) (or their employer(s)) 2019. Re-use permitted under CC BY-NC. No commercial re-use. See rights and permissions. Published by BMJ.

For numbered affiliations see end of article.

Correspondence to Dr Deirdre Hayes-Ryan; deirdre.hayesryan@ucc.ie

\section{ABSTRACT}

Introduction Women presenting with suspected pre-eclampsia are currently triaged on the basis of hypertension and dipstick proteinuria. This may result in significant false positive and negative diagnoses resulting in increased morbidity or unnecessary intervention. Recent data suggest that placental growth factor testing may be a useful adjunct in the management of women presenting with preterm pre-eclampsia. The primary objective of this trial is to determine if the addition of placental growth factor testing to the current clinical assessment of women with suspected preterm pre-eclampsia, is beneficial for both mothers and babies.

Methods and analysis This is a multicentre, stepped wedge cluster, randomised trial aiming to recruit 4000 women presenting with symptoms suggestive of preterm pre-eclampsia between 20 and $36+6$ weeks' gestation. The intervention of an unblinded point of care test, performed at enrolment, will quantify maternal levels of circulating plasma placental growth factor. The intervention will be rolled out sequentially, based on randomisation, in the seven largest maternity units on the island of Ireland. Primary outcome is a composite outcome of maternal morbidity (derived from the modified fullPIERS model). To ensure we are not reducing maternal morbidity at the expense of earlier delivery and worse neonatal outcomes, we have established a co-primary outcome which will examine the effect of the intervention on neonatal morbidity, assessed using a composite neonatal score. Secondary analyses will examine further clinical outcomes (such as mode of delivery, antenatal detection of growth restriction and use of antihypertensive agents) as well as a health economic analysis, of incorporation of placental growth factor testing into routine care. Ethics and dissemination Ethical approval has been granted from each of the seven maternity hospitals involved in the trial. The results of the trial will be presented both nationally and internationally at conference and published in an international peer-reviewed journal.
Strengths and limitations of this study

Randomised trial.

Multiple sites with wide geographic distribution.

- Stepped wedge design.

- Placental growth factor testing only in the intervention arm.

Trial registration number NCT02881073.

\section{BACKGROUND}

Pre-eclampsia is characterised by hypertension and proteinuria, complicates 2\%-8\% of pregnancies, and is associated with significant maternal and neonatal morbidity and mortality. ${ }^{1}$ Currently, women who present with suspected pre-eclampsia are triaged on the basis of hypertension and dipstick proteinuria. Both of these clinical end points are subject to observer error and poor test accuracy, with false positive and negative diagnoses of pre-eclampsia occurring in clinical practice. $^{2-5}$ Current biochemical tests are imperfect at stratifying women for more intensive surveillance as they only identify advanced disease where there is already marked end-organ damage. ${ }^{6}$ While biomarkers and imaging techniques have been evaluated for improving detection, none has adequate sensitivity and/or specificity for the diagnosis of pre-eclampsia. ${ }^{7}$

Placental growth factor (PlGF) belongs to the vascular endothelial growth factor (VEGF) family and represents a key regulator 
of angiogenic events in pathological conditions. ${ }^{8}$ PlGF exerts its biological function through the binding and activation of the receptor Flt-1. ${ }^{9} 10$ In pre-eclampsia, it is thought that endothelial dysfunction leads to an increased level of a circulating decoy receptor, known as soluble Flt-1, (sFlt-1), a soluble receptor for both vascular endothelial growth factor type A (VEGF-A) and PlGF. ${ }^{11}$ Circulating levels of sFlt- 1 are increased in pre-eclampsia and particularly in the early onset form of the disease, resulting in reduced levels of free VEGF-A and PIGF in the maternal circulation. Thus, the endothelial dysfunction observed in pre-eclampsia may be due to excess neutralisation of VEGF-A and PIGF by circulating sFlt-1. Levine et $a l$ showed that in normal pregnancy, PIGF levels track the development of the placenta, peaking at about 32 weeks' gestation when the placenta is developed fully and then declining until delivery. ${ }^{12}$ However, in pre-eclampsia, this rise and fall is considerably lower throughout pregnancy, and levels are strikingly lower when the condition presents clinically.

The PELICAN study (Plasma Placental Growth Factor in the Diagnosis of Women with Pre-Eclampsia Requiring Delivery Within 14 Days:) was the first and largest prospective evaluation of PIGF in women presenting with suspected pre-eclampsia. ${ }^{13}$ This blinded observational cohort study was conducted in seven consultant-led maternity units in the UK and Ireland between January 2011 and February 2012. It enrolled women being investigated for suspected pre-eclampsia, quantified their plasma PlGF using a point of care device, the Alere Triage PIGF test, but did not reveal the result to their clinician. The study found that a PlGF value $<100 \mathrm{pg} / \mathrm{mL}$, in women presenting prior to 35 completed weeks' gestation had a negative predictive value of $98 \%$ (95\% CI 93 to $99.5)$ and a positive predictive value of $44 \%$ (95\% CI 36 to 52) in determining those that would require delivery for a confirmed diagnosis of pre-eclampsia within the next 14 days. The study reported a PlGF $<100 \mathrm{pg} / \mathrm{mL}$ to be a better predictor than all other current commonly used predictive tests of pre-eclampsia, either singly or in combination (blood pressure, urinalysis or biochemical markers) with an area under the receiver operating characteristic curve for low PlGF of 0.87 compared with 0.76 for the next best predictor.

The PROGNOSIS study (Predictive value of the sFlt1:PlGF ratio in women with suspected preeclampsia) was a prospective, multicentre, blinded, observational study conducted in 14 countries from 2011 to $2014 .{ }^{14}$ Its aim was to derive and validate a ratio of serum sFlt- 1 to PlGF that would be predictive of the absence or presence of pre-eclampsia in the short term. It included women with singleton pregnancies from 24 weeks to $36+6$ weeks' gestation in whom a clinical suspicion of pre-eclampsia existed. The Elecsys immunoassay was used to quantify levels of PlGF and sFlt-1. The development cohort of over 500 participants identified a sFlt-1:PlGF ratio of 38 as having an important predictive value. The subsequent validation cohort, again with over 500 participants, reported a negative predictive value of $99.3 \%$ (95\% CI 97.9 to 99.9 ) for ruling out pre-eclampsia within 1 week. Interestingly, the same cut-off of 38 was predictive of the absence of fetal adverse outcomes within 1 week; negative predictive value of $99.3 \%$ (95\% CI 97.9 to 99.9 ). The study showed that an sFlt-1:PlGF ratio of 38 or lower can be used to predict the short-term absence of pre-eclampsia and adverse fetal events in women in whom the syndrome is suspected clinically. ${ }^{15}$ The positive predictive value, a diagnosis of pre-eclampsia, eclampsia or the HELLP syndrome (haemolysis, elevated liver enzymes and a low platelet count) within 4 weeks, was $36.7 \%$ (95\% CI 28.4 to 45.7$)$ using the same sFlt-1:PlGF ratio of 38. Post hoc analysis however showed this was still an improvement in prediction compared with the use of clinical variables such as blood pressure and urinalysis alone.

The National Institute for Health and Clinical Excellence, UK (NICE) has recently published guidance on incorporation of PIGF testing, in addition to clinical assessment, in women presenting with suspected pre-eclampsia from 20 to $34+6$ weeks' gestation. It advises that the Triage PlGF test or Elecsys immunoassay sFlt-1/ PlGF ratio test may be used, in combination with clinical assessment, to 'rule-out' pre-eclampsia in this group of women. However, it advises that these tests should not yet be used to diagnose pre-eclampsia until further research is available, specifically on how an abnormal PIGF result would affect management decisions regarding timing and gestation of delivery and the outcomes associated with this. ${ }^{16}$

The objective of this randomised trial is to evaluate the impact of knowledge of PIGF measurement on clinically relevant outcomes. We hypothesise that adding PlGF measurement to current clinical assessment of women with suspected pre-eclampsia prior to 37 weeks' gestation will reduce associated maternal morbidity through improved risk stratification, earlier diagnosis and targeted management of women with the disease. Any intervention in late pregnancy may have an impact on the fetus. On the one hand, earlier diagnosis of pre-eclampsia may precipitate earlier delivery and lead to an increase in neonatal morbidity and mortality secondary to iatrogenic prematurity. Conversely, improved identification of those neonates at highest risk of imminent placental dysfunction may reduce neonatal morbidity by allowing for timely intervention. It is therefore imperative that full evaluation of both potential benefit and harm is conducted before PIGF testing is implemented routinely into clinical practice. If this trial demonstrates a beneficial impact on maternal morbidity and/or neonatal morbidity, alongside a favourable health economic assessment, then there would be a strong case for incorporating PIGF testing into routine clinical investigations for women presenting with suspected pre-eclampsia before 37 weeks' gestation in a wide variety of healthcare settings. 
Each Cluster $=$ Maternity Hospital

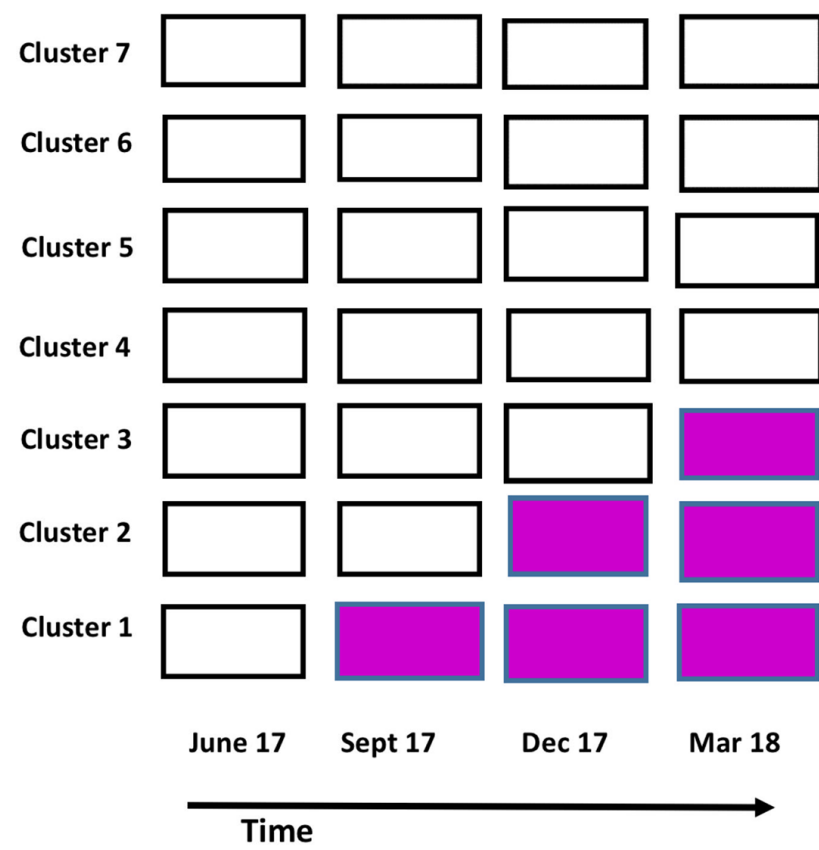

Each Cell = Time Interval

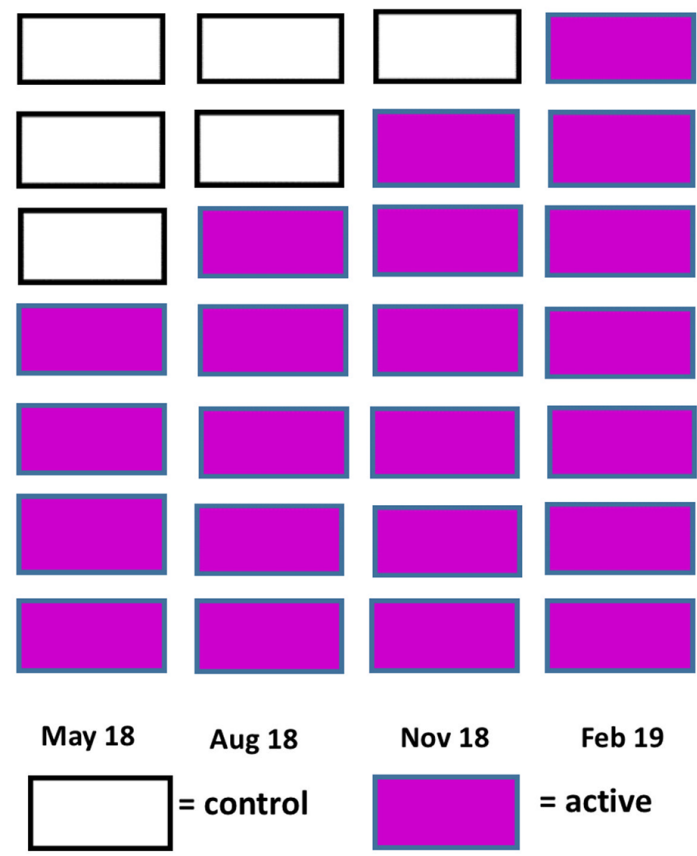

\section{METHODS AND DESIGN \\ Study design}

PARROT Ireland is a multicentre, stepped wedge cluster-controlled trial of PlGF measurement in women presenting with suspected pre-eclampsia from 20 weeks and prior to 37 weeks' gestation. As implementation of a diagnostic test may alter physician management, a cluster design was chosen rather than individual randomisation. This allows for a change in management to occur at a hospital rather than at an individual woman level, which is preferable in trials involving a diagnostic test and allows the clinical influence of the additional test to be evaluated in a pragmatic fashion. ${ }^{17}$ Each maternity hospital acts as a cluster. All clusters commenced the trial in the control arm and in turn, each cluster transitions at random from the control to the intervention at prespecified time points. Once a cluster has changed over to the intervention, it continues as such for the remainder of the trial so that by the end of the trial all clusters will be in the intervention arm (figure 1). A stepped wedge design was chosen so as to increase the social acceptability of the trial to the seven hospitals (the stake holders/decision makers in all of the hospitals expressed a desire to participate in a trial in which they were guaranteed to get the intervention); and because a trial with just seven clusters risks baseline imbalance in a parallel design.

The trial will continue for a period of 22 months, and with seven clusters the interval between transitions is approximately 3 months in duration. A restricted method of randomisation was used to provide a balance in total (expected) number of observations across intervention and control periods (details below). ${ }^{18-20}$ There is a short transition period of 1 week whenever a new cluster transitions from control to the intervention. Data collected during this transition period will not be included in any analysis of outcomes. Recruitment will stop on a prespecified fixed date in late April 2019 and the study will end when the last recruited participant and neonate are discharged and all outcome data collected.

\section{Setting and participants}

The trial is being conducted within the Health Research Board Mother and Baby Clinical Trial Network Collaborative. The Coombe Women and Infants University Hospital Dublin, Cork University Maternity Hospital, University Maternity Hospital Limerick, The Royal Jubilee Maternity Hospital Belfast, University College Hospital Galway, The National Maternity Hospital Dublin and The Rotunda Maternity Hospital Dublin are the seven largest consultant-led maternity units on the island of Ireland. Combined, they have an annual birth rate of over 44000 , representing over half of the country's total annual births. Women attending these maternity units who present with suspected preterm pre-eclampsia are eligible for inclusion in this trial. Detailed inclusion and exclusion criteria are described in boxes 1 and 2 .

\section{Randomisation}

The trial statisticians for the study developed a randomisation sequence for site transition from control to intervention; however, the order of site transitioning is concealed from sites and principal investigators until 12 weeks prior to the sites transition date. An allocation sequence was randomly selected (ie, a cross-over order for the seven clusters) from a set of random sequences constrained so that the sum of the total cluster sizes in 


\section{Box 1 Inclusion criteria}

Pregnant women between $20+0$ and $36+6$ weeks of gestation (inclusive) with a;

- Singleton pregnancy.

- Aged 18 years or over.

- Able to give informed consent.

- Presenting with suspected pre-eclampsia: (one or more of the following):

- Hypertension;

- Dipstick proteinuria;

- Headache;

- Visual disturbances;

- Epigastric or right upper quadrant pain;

- Increasing oedema;

- Suspected fetal growth restriction;

- If the healthcare provider deems that the woman requires further evaluation for possible pre-eclampsia.

the intervention status was similar to the total sum of the cluster sizes in the control status. Similar was defined to be a difference in the total sums exposed to intervention and control statuses being no different than the expected middle 25th percentile range of differences. To implement this, 10000 simulations of possible (unique) allocation sequences were performed. From this, the difference in number exposed to intervention and control for each sequence was determined. An allocation sequence was then selected at random from those falling within the middle 25th percentile range of differences. ${ }^{17-19}$

\section{Control}

Eligible women are approached and provided with detailed information about the trial, both verbally and written, by a trained researcher. Eligibility is determined by review of symptoms and signs at the time of presentation to the maternity hospital by the local researcher. Participants are not aware of their maternity hospitals

\section{Box 2 Exclusion criteria}

- Confirmed pre-eclampsia at point of enrolment: 'sustained hypertension with systolic blood pressure (BP) $\geq 140$ or diastolic $\mathrm{BP} \geq 90 \mathrm{~mm} \mathrm{Hg}$ on at least two occasions (at least 4 hours apart) with significant quantified proteinuria (>300 mg protein on 24 hours collection or urine protein creatinine ratio $>30 \mathrm{mg} / \mathrm{mmol}$ ) or abnormal pre-eclampsia bloods'.

$>37$ weeks' gestation.

- Multiple pregnancy.

- Abnormal pre-eclampsia bloods (new-onset reduced number of platelets or deranged liver function/renal function tests, identified during routine care prior to enrolment and not attributable to anything other than pre-eclampsia).

- Decision regarding imminent delivery already made.

- Lethal fetal abnormality present.

- Previous participation in PELICAN trial in a prior pregnancy.

- Participation in a conflicting trial at the same time as PARROT Ireland.

Plan to use off protocol placental growth factor testing. current randomisation prior to their enrolment on the trial. Informed consent is obtained in accordance with ICH-GCP guidelines. ${ }^{21}$ Once an eligible woman has given written informed consent for inclusion in the study, her maternity hospitals current group allocation is revealed (figure 2). Participants enrolled in the control arm receive usual hospital care as per National guidelines; these are Health Service Executive/Institute of Obstetrics and Gynaecology Irish guidelines for those in the Republic or the NICE guidelines for those in Northern Ireland (figure 3A,B) ${ }^{22}{ }^{23}$ Eligible women who are approached but who decline to participate in the trial will continue to receive usual hospital care.

\section{Intervention}

Participants enrolled in the intervention arm have their plasma PIGF quantified in addition to routine hospital investigations. The PlGF result is made immediately available to the participants clinical team and documented clearly in the participant's medical notes. A suggested further management algorithm is provided to the clinician based on both the degree of hypertension present and the PlGF result (figure 4). This algorithm advocates increased frequency of review for those participants identified as having an abnormal PIGF result. The final decision regarding frequency of review remains with the treating clinician. If 4 weeks or more pass and the participant represents with symptoms suggestive of pre-eclampsia, a repeat PlGF quantification may be performed as long as the inclusion/ exclusion criteria are still satisfied. In certain sites the option of plasma Biobanking will be available. Participants will be consented separately for this. For those who give consent, a portion of the specimen taken will be used to measure the level of PlGF in the plasma and the remainder of the sample will be stored in University College Cork Biobanking facility.

\section{PIGF quantification}

Maternal plasma PIGF quantification is performed on an EDTA venous blood sample obtained in the standard fashion. Plasma is obtained through centrifugation and the sample is then processed immediately using a $\mathrm{CE}$ marked validated point of care platform; the automated Triage Meterpro (ALERE San Diego, California, USA). Each hospital has the necessary equipment in situ and appropriately trained researchers in place, to perform this test as per manufacturer's guidelines. The PlGF measurement is reported as the absolute value in $\mathrm{pg} / \mathrm{mL}$ within $30 \mathrm{~min}$ of commencing processing of the sample. All samples taken will be analysed without delay by the researcher after venepuncture has occurred and in accordance with manufacturer's instructions. The Triage PlGF test platform and consumables necessary to perform testing are brought to the cluster just at the point of transition to intervention. It is therefore not available at site for use while the site is in the control arm. 


\section{$20+0$ - 36+6 weeks with suspected PET}

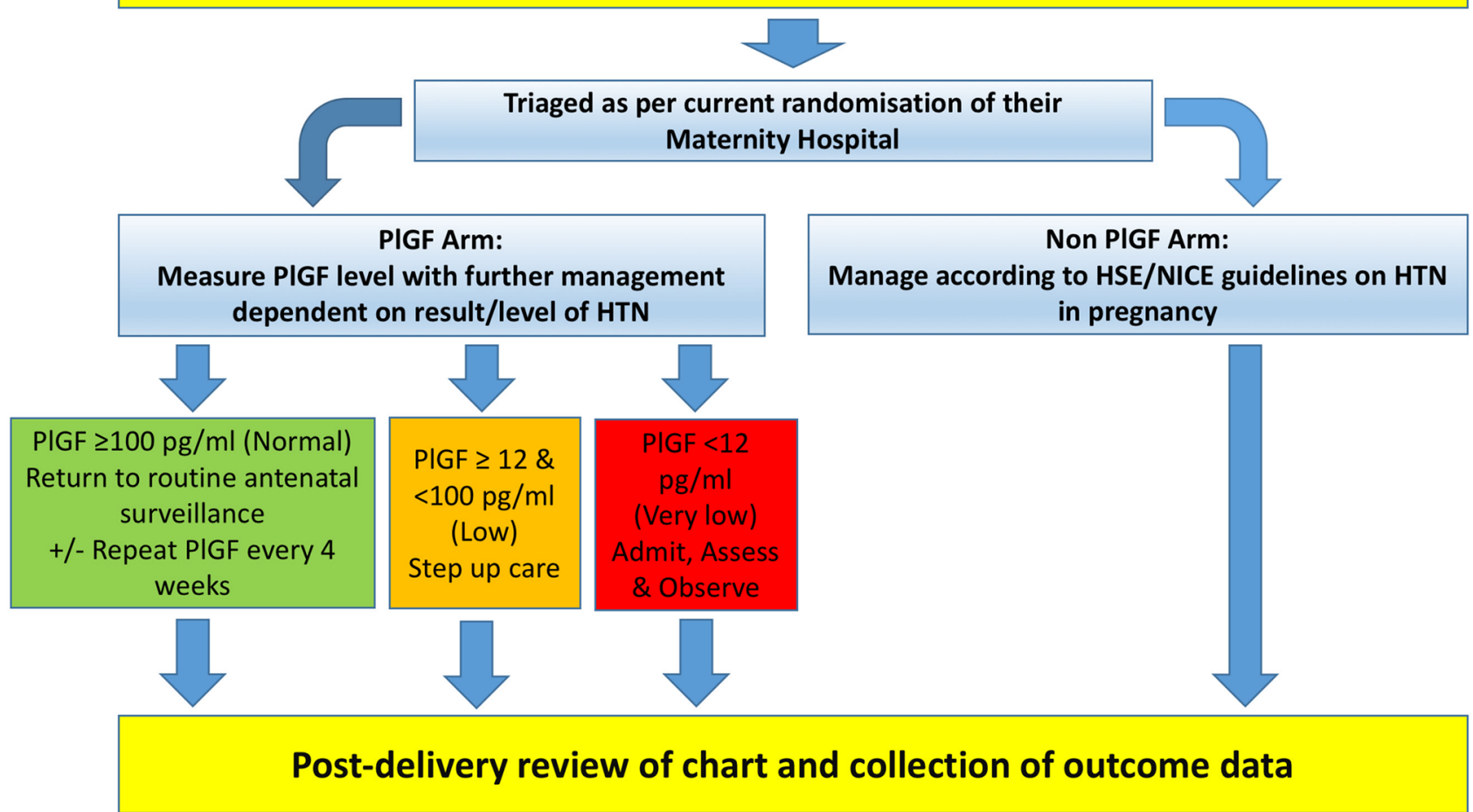

Figure 2 Trial schematic for PARROT Ireland. HTN, hypertension; NICE, National Institute for Health and Clinical Excellence; PIGF, placental growth factor.

\section{Patient and public involvement}

Patients/public were not involved in the development of this trial.

\section{Outcome measure}

\section{Primary outcome measure}

To evaluate if the intervention is beneficial to both women and their babies and more importantly to ensure it is not harmful to either, the study has two equally important co-primary outcome measures. These are maternal morbidity and neonatal morbidity. For maternal morbidity assessment, an adaption of the fullPIERS score is used (box 3). The definition of hepatic dysfunction is based on alanine aminotransferase rather than International Normalised Ratio (INR), requirement for intensive care unit admission is included as well as the presence of severe hypertension. Severe systolic hypertension is an independent risk factor for stroke in pregnancy and in high-resource settings uncontrolled hypertension is the main cause of death in women with pre-eclampsia. ${ }^{24-26}$ The interval from diagnosis of pre-eclampsia to delivery is not a suitable outcome measure to use, as we are aware that knowledge of PIGF result may alter clinician management and expedite delivery. ${ }^{27}$ For neonatal morbidity assessment, babies are dichotomised into having or not having identified neonatal morbidity by means of a composite neonatal score (box 4). In order to avoid subjectivity in the diagnosis of morbidity, the majority of components of the neonatal composite score are objective measures; $\mathrm{pH}<7.2$, positive cultures, admission to NICU. We acknowledge that some subjectivity can arise with staging of disease hence why all stages of each disease will be captured and will comprise the composite outcome; NEC stage 1-3, IVH grade 1-4 and ROP stage $1-5$. Neonatal outcomes and morbidity will be captured from local case note review, as documented by the treating neonatologist. In cases where any uncertainty is present, the researcher will discuss the case with the local Principal Investigator (PI) and or the trial clinical fellow and a consensus will be reached

\section{Secondary outcome measure}

Secondary outcomes include each component of the primary outcome reported individually as well as further maternal and neonatal assessments such as mode of delivery and use of antihypertensive agents (boxes 5 and 6). Fetal growth restriction, identified on antenatal ultrasound, has been included as a secondary outcome measure of neonatal morbidity. As PlGF correlates well with placental dysfunction, it may be able to differentiate between those babies with pathological growth restriction rather than constitutional growth restriction and hence improve neonatal outcomes.

A separate health economic evaluation is assessing the intervention's economic impact. This is achieved through the use of participant quality of life (QoL) questionnaires (EuroQol 5D and Short-Form 36), ${ }^{28}{ }^{29}$ a specially designed study-specific participant costing questionnaire and by assessment of costs to the health service 
A

\section{PARROT $\quad$ Management Algorithm \\ IRELAND PARROT Ireland_Republic}

If patient enroled in CONTROL arm - manage according to degree of hypertension present

\begin{tabular}{c} 
Normotensive or \\
mild hypertension: \\
BP up to $149 / 99 \mathrm{mmHg}$ \\
Test for proteinuria weekly \\
PET bloods at presentation \\
then as per routine clinical \\
care \\
Do not treat BP \\
Fetal US if $<34$ weeks \\
\hline
\end{tabular}

Moderate hypertension: BP 150/100-159/109 mm Hg

Commence BP Treatment Measure $\mathrm{BP}$ and urine at least twice a week (If PCR> 30, do not repeat) PET bloods at presentation then as per routine clinical care Fetal US if $<34$ weeks
Severe hypertension: $B P \geq 160 / 110 \mathrm{mmHg}$

Admit to hospital until BP stabilises

Commence BP Treatment

Measure BP at least $x$ 4/day while inpatient Test for proteinuria

(if $\mathrm{PCR}<30$ check daily and once $>30$ do not repeat)

PET bloods at presentation, repeat at least weekly.

Fetal US, AFI, Doppler \& CTG

Management Algorithm Version $3.025^{\text {th }}$ October 2017

B

\begin{tabular}{|cc}
\hline PARROT & $\begin{array}{l}\text { Management Algorithm } \\
\text { IRELAND }\end{array}$ \\
PARROT Ireland_Belfast
\end{tabular}

If patient enroled in CONTROL arm - manage according to degree of hypertension present

Normotensive or
mild hypertension:
BP up to $149 / 99 \mathrm{mmHg}$
Do not admit/treat BP
Measure BP weekly
Test for proteinuria at each
visit
Only those bloods for
routine antenatal care
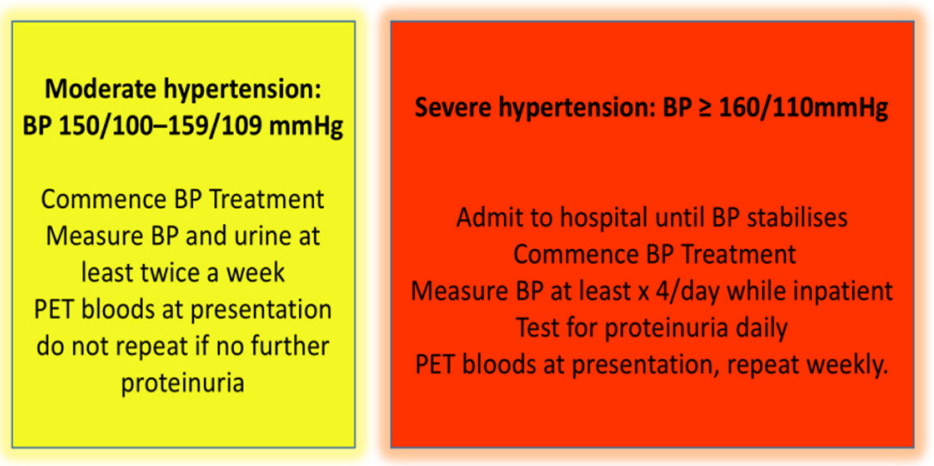

Management Algorithm Version $3.025^{\text {th }}$ October 2017

Figure 3 (A) Management algorithm for control arm based on Health Service Executive (HSE) guidelines for PARROT Ireland. (B) Management algorithm for control arm based on NICE guidelines for PARROT Ireland. AFI, amniotic fluid index; BP, blood pressure; CTG, cardiotocography; NICE, National Institute for Health and Clinical Excellence; US, ultrasound.

of community-based/inpatient/day case care, through chart review at discharge. ${ }^{30-32}$

\section{Data collection}

Trial data captured locally at site by researchers are transmitted securely using an electronic clinical record form (eCRF) to a specific database developed by MedSciNet. Baseline demographic data, QoL questionnaires and the PlGF result are entered live to the eCRF at point of recruitment. The full eCRF is completed after discharge from the maternity hospital postdelivery, and includes neonatal and maternal medical outcome, costing questionnaire and repeat QoL questionnaires. All data entered to the eCRF is pseudo-anonymised with each participant identified by a unique study number. The identifier key is kept separately locally at site in a secure location. The data system is built to the same security and confidentiality standards as those of hospital electronic health records. The data at each participating centre are handled in accordance with local regulatory legislation and ethics committee approval. A detailed description of schedule and timing of data collection is provided in table 1 . 


\section{PARROT IRELAND \\ Management Algorithm PARROT Ireland}

\section{Sample size}

The sample size was fixed by the number of sites and the study duration. It is anticipated that the total sample size will be in the region of 4000 participants; split across seven clusters and the eight time periods in the design (equivalent to a cluster-period size of about 71). With a sample size of 4000 and using a two-sided type I error rate of 0.025 (to allow for two co-primary outcomes), we determined the power to detect a $7 \%$ reduction in maternal

\section{Box 3 Components of the maternal morbidity composite}

\section{score}

- Confirmed placental abruption.

- Intensive care admission.

- Central nervous system compromise:

- Generalised tonic clonic seizure due to eclampsia, Glasgow Coma Scale $($ GCS $)<13$, cerebral haemorrhage/infarct, cortical blindness, retinal detachment, transient ischaemic attack, reversible ischaemic neurological deficit.

- Cardiorespiratory compromise:

- Myocardial ischaemia/infarction, $\quad \mathrm{SpO}_{2}<90 \%, \quad>50 \%$ FiO for $>1$ hour, intubation (other than for caesarean section), pulmonary oedema, need for positive inotrope support.

- Haematological compromise:

- Transfusion of any blood product, platelet count $<100 \times 10^{9} / \mathrm{L}$.

- Liver compromise:

- Hepatic dysfunction (alanine aminotransferase or aspartate aminotransferase $>70 \mathrm{IU} / \mathrm{L}$, haematoma, rupture.

- Kidney compromise:

- Acute renal insufficiency (creatinine $>150 \mu \mathrm{mol} / \mathrm{L}$ ); haemodialysis.

- Severe hypertension:

- Systolic blood pressure $\geq 160 \mathrm{~mm} \mathrm{Hg}$ on at least one occasion. morbidity (relative risk reduction of 20\%) from $35 \%$ to $28 \%$ in the intervention, that is, 'active' group (based on a reported rate of adverse maternal outcome in the region of $35 \%$ in the PELICAN trial). ${ }^{1333}$ This is assuming an intraclass correlation (ICC) in the region of 0.01 ; and consider sensitivity to a range of intracluster correlation (ICC) values between 0.005 and 0.05 . The second co-primary outcome is adverse neonatal outcomes. Due to scarcity of information on the ICC, the same ICC as for the maternal outcome is assumed. Current rates of adverse events are around $10 \%$. We determine power to detect an absolute change in neonatal adverse outcomes of $6 \%$.

To allow for the longitudinal nature of the trial, where correlations may differ between observations in the same

\section{Box 4 Components of the neonatal morbidity composite}

\section{score}

- Perinatal death or death before hospital discharge.

- Neonatal intensive care unit admission for $\geq 48$ hours.

- Birth weight $\leq 5$ th customised centile*.

- Apgar score $<7$ at $5 \mathrm{~min}$.

- Umbilical artery acidosis at birth (cord $\mathrm{pH}<7.2)$.

- Admission to neonatal unit.

- Respiratory distress syndrome.

- Interventricular haemorrhage (IVH).

- Retinopathy of prematurity (ROP).

- Confirmed infection (confirmed on blood or cerebrospinal fluid cultures).

- Necrotising enterocolitis (NEC).

${ }^{*}$ Customised birth weight at delivery is calculated using the Gestation Related Optimal Weight (GROW) centile. 


\section{Box 5 Secondary outcomes-maternal}

- Final diagnosis of hypertensive disorder of pregnancy (chronic hypertension (HTN), gestational HTN or pre-eclampsia).

- Gestation at diagnosis of pre-eclampsia.

- Use of one or more antihypertensive drugs.

- Instrumental delivery (ventouse or forceps):

- Severe HTN (systolic blood pressure (BP) $\geq 160 \mathrm{~mm} \mathrm{Hg}$ on at least one occasion);

- Maternal morbidity by fullPIERS model;

- Confirmed placental abruption;

- Intensive care admission;

- Central nervous system compromise;

- Cardiorespiratory compromise;

- Haematological compromise;

- Liver compromise;

- Kidney compromise.

- Progression to severe pre-eclampsia as defined by the American College of Obstetricians and Gynecologists practice bulletin:

- Systolic BP $\geq 160 \mathrm{~mm} \mathrm{Hg}$ or diastolic $B P \geq 110 \mathrm{~mm} \mathrm{Hg}$ on two occasions at least 4 hours apart while the patient is on bed rest (unless antihypertensive therapy is initiated before this time);

- Thrombocytopenia (platelet count<100×109/L);

- Impaired liver function as indicated by abnormally elevated blood concentrations of liver enzymes (to twice normal concentration), severe persistent right upper quadrant or epigastric pain unresponsive to medication and not accounted for by an alternative diagnoses, or both

- Progressive renal insufficiency (serum creatinine concentration $>1.1 \mathrm{mg} / \mathrm{dL}(150 \mu \mathrm{mo} / \mathrm{L})$ or a doubling of the serum creatinine concentration in the absence of other renal disease);

- Pulmonary oedema;

- New-onset cerebral or visual disturbances.

- Elective delivery: induction of labour or caesarean section.

Caesarean section: emergency and elective

cluster-period; and those measured in different cluster periods, we incorporate cluster-autocorrelations (CAC). There is little information to support likely values for the CAC, so we are guided by values in the literature and explore sensitivity across a range of values $(0.64,0.80$ and 0.96). ${ }^{3435}$

The power has been estimated using an online RShiny App. ${ }^{36}$ We have not included transition periods in the calculation but given the transition periods are just 1 week in length, this is not expected to significantly affect power. There has been no allowance for varying cluster sizes as this is currently not something which is technically possible in a stepped wedge study. Sample size calculations were performed assuming linear mixed models with categorical effects for time; random cluster and random cluster by period effects. ${ }^{38}$ Under these assumptions, we constructed power curves, which reveal that under most anticipated scenarios the trial will have in the region of $80 \%$ power (figures 5 and 6 ). ${ }^{35} 39$

\section{Data analysis}

\section{Clinical outcome}

The primary aim of the study is to evaluate whether there is a difference in the two composite outcomes before

\section{Box 6 Secondary outcomes-neonatal}

- Fetal growth restriction identified on antenatal ultrasound* (estimated fetal weight and/or abdominal circumference $<10$ th customised centile, abnormality in umbilical artery Doppler velocity or reduced level of amniotic fluid).

- Gestation at delivery.

- Perinatal death or death before hospital discharge.

- Admission to neonatal intensive care unit (NICU).

- NICU admission for $\geq 48$ hours.

- Birth weight $\leq 5$ th customised centile.

- Apgar score $<7$ at $5 \mathrm{~min}$.

- Umbilical artery acidosis at birth (arterial cord pH<7.2).

- Respiratory distress syndrome.

- Interventricular haemorrhage.

- Retinopathy of prematurity.

- Confirmed infection (confirmed on blood or cerebrospinal fluid cultures).

- Necrotising enterocolitis.

*Antenatal detection of fetal growth restriction is based on formal ultrasound assessment of fetal biometry using the Hadlock formula.

and after exposure to the intervention. There will be no double counting of outcomes, individuals not events will be presented for the composite. Mixed effects regression models will be used to allow for the clustering within sites. Calendar time will also be adjusted for since the intervention is sequentially rolled-out both by including fixed categorical time effects and random cluster by categorical time effects. $^{40}$

The primary estimate of the treatment effects will therefore be cluster and time adjusted. Time adjustment is essential, as it is a stepped wedge trial. Log Poisson regression models with robust variance estimation (to allow for misspecification of binomial errors) will be used so as to allow estimates of relative risks ${ }^{41}$; to estimate risk differences corresponding Binomial models with log links will be fitted. Secondary analysis will adjust for individual and cluster level covariates. In the first instance, comparative estimates of differences between groups will be adjusted for variables used in the randomisation procedure (eg, site, time and hospital size). Furthermore, more fully adjusted analyses will also be performed. These more fully adjusted analyses will adjust for gestational age at recruitment, maternal age, smoking status, maternal body mass index, public versus private obstetric care and maternal comorbidities such as chronic renal disease, systemic lupus erythematosus (SLE), antiphospholidip syndrome (APS) and diabetes. It will also adjust for hospital size $(<5000$ or $>5000$ deliveries/ annum). Categorised continuous variables (eg, age) will be treated as continuous variables in this adjustment. If covariate adjustment is not practical, unadjusted estimates will be produced and it will be made clear in the output why this occurred (eg, not possible due to low event rate lack of model convergence). Null hypotheses and analyses for secondary outcomes take a similar form to that for the primary outcome, and where outcomes are not binary, analysis will be using the generalised linear mixed model. 
Table 1 Standard Protocol Items: Recommendations for Interventional Trials flow diagram for schedule of events in PARROT Ireland

\section{On presentation with suspected PET Between $20+0$ and $36+6$ weeks}

In-person visit
From enrolment to discharge postdelivery

\section{Discharge \\ postdelivery}

In-person

Chart In-person visit Chart completed

$\begin{array}{llll} & & & \\ \text { Randomisation-institutional level } & \mathrm{X} & & \\ \text { Inclusion/exclusion } & \mathrm{X} & \mathrm{X}^{*} & \\ \text { Informed consent } & \mathrm{X} & \mathrm{X}^{*} & \mathrm{X} \\ \text { Demographics } & & \mathrm{X}^{*} & \\ \text { History, comorbidities } & & \mathrm{X}^{*} & \\ \text { Con medications } & & \mathrm{X}^{*} & \\ \text { Physical measurements } & & & X \neq \\ \text { Clinical readings } & \mathrm{X} & & \end{array}$

\begin{tabular}{|c|c|c|c|c|}
\hline PIGF† measurement & $x$ & $x \neq$ & & \\
\hline Biobank sample§ & $x$ & & & \\
\hline Fetal assessments & & & $x$ & \\
\hline Prenatal admissions & & & $x$ & \\
\hline Maternal PET bloods & & & $\mathrm{x}$ & \\
\hline Newborn data & & & $\mathrm{x}$ & \\
\hline Neonatal outcome & & & $x$ & \\
\hline Maternal outcome & & & $x$ & \\
\hline Complications & & & $x$ & \\
\hline Postnatal admissions & & & $x$ & \\
\hline Clinical management & & & $x$ & \\
\hline Final outcomes & & & $x$ & \\
\hline EQ-5D, SF-36 & $\mathrm{x}$ & & & $x$ \\
\hline Costing questionnaire & & & & $x$ \\
\hline In-person visits & $\mathrm{x}$ & $x \neq$ & & \\
\hline
\end{tabular}

*May be captured in chart review or in consultation with participant at any time following enrolment.

†PIGF testing depends on institutional randomisation allocation.

‡PIGF testing will be repeated if readmission for suspected pre-eclampsia. May be repeated more than once. No more often than four weekly. §Only at biobanking sites.

EQ-5D, EuroQol 5D; PIGF, placental growth factor; SF-36, Short-Form 36.

Transformations will be performed where data are markedly not normally distributed. For the analysis adjusted for covariates and for the secondary outcomes (unadjusted), multiple imputation methods will be used if the proportion of missing data is $>5 \%$, and this multiple imputation will also allow for the clustered and temporal nature of the trial. It is not expected that there will be any missing data in the primary outcome, as it will be assumed that if the outcome is present then it will be recorded and if it is not recorded we will assume it is absent. This is a standard and realistic assumption. Results will be presented as adjusted risk ratios with CIs and risk differences to allow full appreciation of clinical effect. To allow for the two primary outcomes, we will follow good practice and adjust for this multiplicity using a Bonferroni correction and so report 97.5\% CIs.

For secondary continuous outcomes mean differences will be reported and $99 \%$ CIs for secondary outcomes. We will report latent ICCs for all outcomes, along with 95\% CIs. Prespecified subgroup analysis will be undertaken on the primary outcome based on women presenting $<35$ weeks' gestation vs $>35$ weeks' gestation; size of unit and final confirmed diagnosis. The stepped wedge trial design will also allow investigation of treatment effect heterogeneity across clusters and time. These exploratory analyses will be reported using 99\% CIs. Analysis will be conducted by intention to treat and sites will be considered exposed to the intervention postrandomised cross-over date.

Health economic outcome

The economic evaluation will be informed by a decision analytical model, which will be designed and constructed for the study to reflect the maternal and fetal pathway and health states. Employing a decision analytical model allows for the extrapolation of existing data and the opportunity 


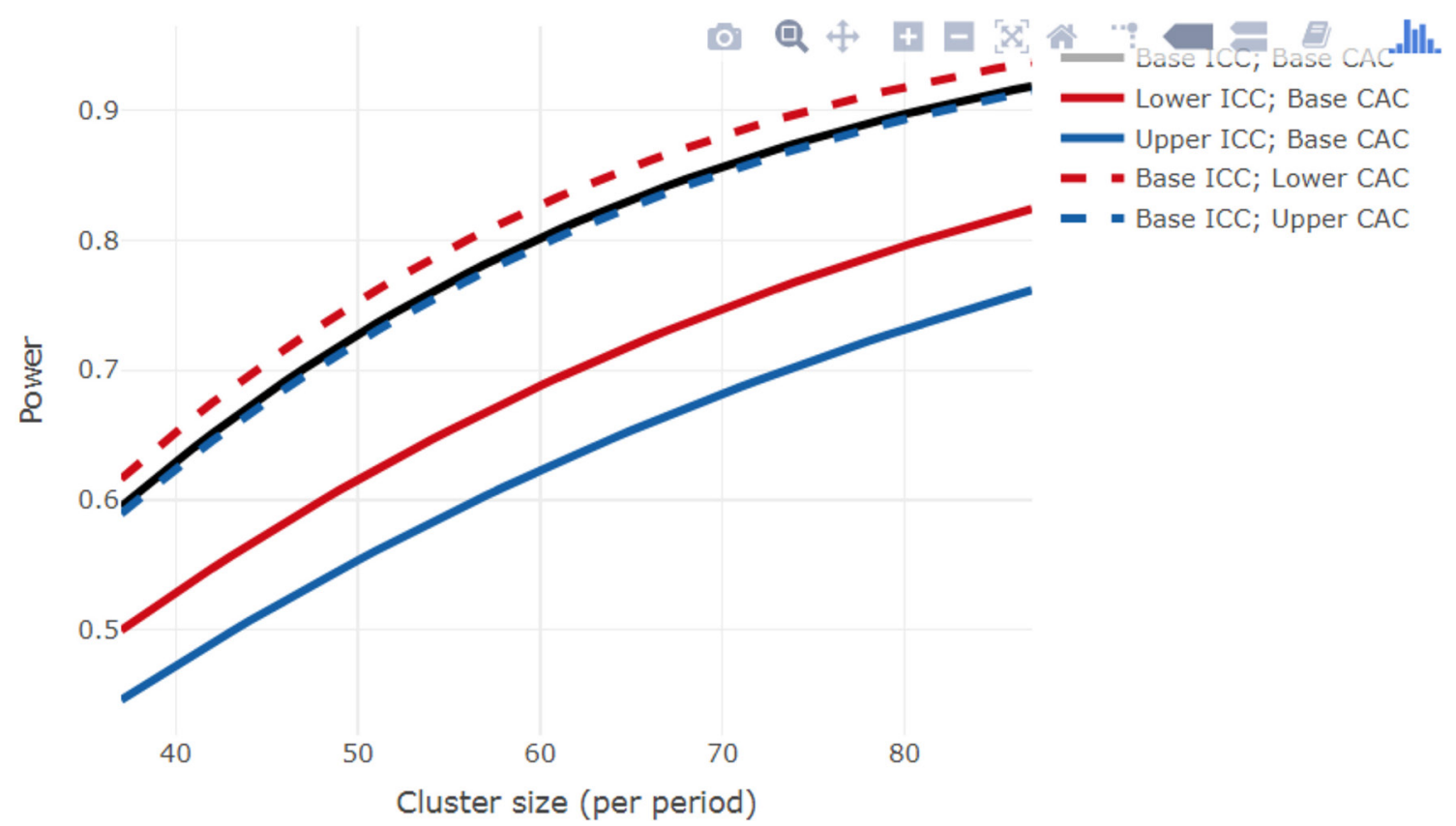

Figure 5 Power curve for PARROT Ireland for maternal adverse outcomes. CAC, cluster-autocorrelation; ICC, intracluster correlation.

to systematically synthesise evidence from various sources. Primary data on maternal health outcomes will be available from the study with the distribution of 5-level EQ-5D version and SF-36 questionnaires which will inform the estimation of quality-adjusted life years (QALYs). Neonatal outcomes will be informed by secondary sources. A systematic literature review will be conducted to identify QOL/utilities (or proxies for same) associated with neonate outcomes, which will be incorporated into the decision analytical model to estimate QALYs. Primary data on resource utilisation will be collected using the costing questionnaire. The costs and effects of the intervention and comparator will be compared with an incremental cost-effectiveness ratio in a cost utility analysis. To address parameter and structural uncertainties, a probabilistic sensitivity analysis will be performed.

\section{Trial management}

Day-to-day running of the trial will be coordinated by the Trial Management Group (TMG). The TMG consist of the lead site investigator plus the project manager and the clinical fellow. The TMG will act on behalf of the sponsor and will be responsible to the Trial Steering

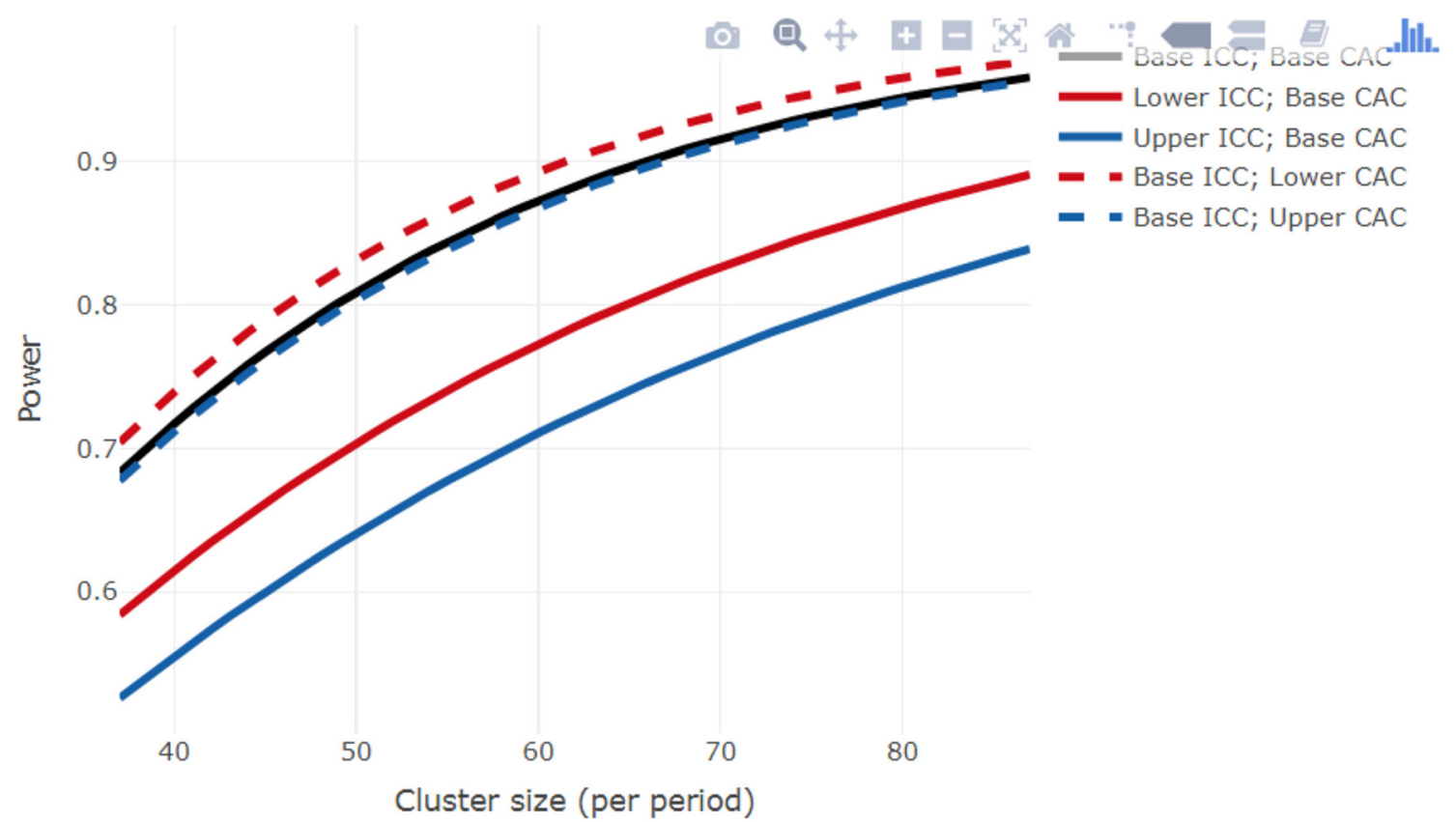

Figure 6 Power curve for PARROT Ireland for neonatal adverse outcomes. CAC, cluster-autocorrelation; ICC, intracluster correlation. 
Committee (TSC) to ensure that all sponsors' responsibilities are carried out. The TSC comprises all PIs as well as the TMG, sponsor, Health Research Board (HRB) and representatives from statistics, economics, neonatology, laboratory and a lay person. The role of the TSC is to provide overall supervision of the trial. In particular, the TSC will concentrate on the progress of the trial, adherence to the protocol, participant safety and consideration of new information.

\section{Data monitoring}

To provide protection for study participants an independent data monitoring committee (DMC) has been appointed for this trial. The DMC comprises four members who are not involved with any other aspect of the trial. They include an obstetrician, a neonatologist, a statistician and a midwife. The DMC met and ratified their charter and have advised that all serious adverse events such as stillbirth/neonatal death or profound maternal morbidity in the Intervention arm of the study be reported to them immediately. The DMC will receive regular updates on the progress of the trial every quarter from the TMG. The purpose of these updates is for the DMC to: 1) ensure the quality of data collection; 2) ensure that the intervention is being rolled out according to the randomisation plan; 3) monitor balance between arms to monitor for potential selection biases and 4) ensure PIGF testing is not overwhelmingly better or worse than no PlGF testing with respect to maternal morbidity with neonatal morbidity. Once 1500 outcomes are available an interim analysis will be conducted and reviewed by the DMC. The interim analysis will report on the co-primary outcomes, follow the same methods as those of the primary analysis and examine if there is proof beyond reasonable doubt that one particular intervention is definitely indicated or definitely contraindicated in terms of a net difference of a major end point. There will be no formal stopping criteria put in place, but the DMC will be guided by the knowledge that proof beyond reasonable doubt cannot be specified precisely, but a difference of at least three SD in an interim analysis of the primary outcome would be consistent with strong level of evidence. No allowance for this interim analysis has been made in power calculations.

There will be no stopping of the trial for futility as the study will be underpowered to detect small effects.

\section{DISCUSSION}

Based on previous experience during the PELICAN study, an analysis of success criteria and barriers to our proposed study was conducted. Potential barriers include the overestimation of (i) identification of eligible women by the research team, (ii) primary outcome event rate (iii) and retention/attrition, that is, gaining outcomes data on all women included.

A recruitment feasibility audit conducted in Cork University Maternity Hospital (CUMH) over the course of a typical week in July 2016 identified 21 women who would be eligible for inclusion in the PARROT Ireland study. This would equate to almost 1100 women per annum in CUMH, approximately $13 \%$ of its annual delivery rate. This is in keeping with the quoted $10 \%$ incidence of hypertensive disorders of pregnancy in the population. ${ }^{42}$ It is anticipated that over the 22-month duration of the study across the 7 hospitals approximately 10486 women will meet the study inclusion criteria (13\% of the combined annual delivery rate), and of these 4000 will be recruited into this trial (approximately 38\% of those eligible). As inclusion in the trial will be optional and require informed consent from participants, not all eligible women in each unit will be included. Projected inclusion rates will be apparent via a dedicated MedSciNet database pre-programmed, available online and contemporaneously updated, allowing prompt action to intervene when not optimal. A conservative requirement of $<50 \%$ of all eligible women to be recruited in order to reach targets has deliberately been chosen and successful recruitment of the same population in the PELICAN study is reassuring. As with any study we may get a higher or lower incidence of the primary outcome of interest than anticipated. We should get an early indication of this at the interim analysis.

As participation in the trial does not require any extra attendances/input from the participant for the remainder of the pregnancy, it is likely that retention of participants will not be an issue. Similarly, the data outcome to assess for maternal and neonatal morbidity can be readily obtained postdelivery following discharge of the participant from their stored medical records locally at each unit. However, in order to fully examine the health economic outcomes there exists a reliance on the return of completed questionnaires by the participant postdelivery. To minimise attrition rates, the researcher at each site will endeavour to meet with each participant postdelivery prior to their discharge and encourage them to complete the health economic questionnaires. In the PELICAN study, only $1 \%$ of the cohort were lost to follow-up. The risk of incomplete data collection of outcomes in studies such as this is more relevant if women deliver in a different unit to that which they are recruited into the trial. However, all seven clusters in our trial are large tertiary referral units and patient transfer during pregnancy is rare. We are therefore confident that the likely rate of loss to follow-up will be similar and in the order of $1 \%$.

There are a number of advantages with the use of stepped wedge design. It allows a phased implementation of the intervention, which is preferable when commencement in all clusters simultaneously would be challenging. As all clusters ultimately receive the intervention, it increases willingness of the clusters to partake in the trial. We acknowledge that seven clusters is a small number of clusters and this is an important limitation of the study. Mostly, this is a limitation because it will mean that the findings have questionable generalisability. But, if these clusters are representative then the findings may still be 
generalisable in part. The other limitation that seven clusters brings about is questionable internal reliability. However, because all of the clusters receive both the intervention and control condition, the clusters serve as their own controls. This lessens the impact of chance imbalance and it increases the power of the study (particularly so when the ICC is large, as is the case here). The study does only have in the region of $80 \%$ power and should parameters such as the ICC be very different to that which we have assumed, then it is correct that the study might be underpowered. To ensure that this is properly accounted for at the analysis stage, we will report appropriate CIs around all point estimates, so the impact of any impression is properly reported.

Another potential limitation worth noting is the slightly different management algorithm for one cluster, Belfast, in the control arm. The Belfast control arm algorithm is taken directly from the NICE Hypertension in Pregnancy guidelines. All other clusters are using an algorithm taken from the HSE guidelines for Hypertension in Pregnancy. The two are essentially the same except the HSE algorithm also includes a recommendation for a fetal ultrasound in cases where the participant is $<34$ weeks' gestation. It is not anticipated that the difference in these algorithms should have any bearing on the overall trial results. We will conduct a sensitivity analysis with the Belfast site removed and see if the result remains consistent.

Ideally, PIGF testing should be performed for all participants enrolled in the study, with blinding of the result for those in the control arm. This would allow for test performance statistics to be performed. Unfortunately, testing of control participants will not be conducted in our trial, which is a notable limitation of the study.

The primary aim of the PARROT Ireland trial is to establish the effectiveness of revealed plasma PlGF measurement in reducing maternal morbidity (with assessment of neonatal safety in parallel) in women presenting with suspected pre-eclampsia prior to 37 weeks' gestation. Should the trial show a reduction in maternal morbidity without an increase in neonatal morbidity, or indeed a reduction in neonatal morbidity with no change in maternal morbidity, it would provide a strong argument for its incorporation into routine obstetric practice. The long-term aim of the trial is to demonstrate if PlGF measurement enables appropriate antenatal stratification of women presenting with suspected pre-eclampsia.

Avoiding unnecessary hospital admission would be both clinically and economically beneficial. In contrast, those at increased risk of imminent adverse events, identified by an abnormal PlGF result, would have hospital resources redirected to them. We anticipate that this trial will provide a definitive result on the benefits of PlGF testing, which will act to influence international clinical practice.

A separate randomised controlled trial (RCT), also entitled 'PARROT', has completed recruitment in the UK since the end of 2017. Although recruiting a similar population of women and using the same PlGF platform, the primary outcome measure for the two RCTs is different, with the UK PARROT trial focusing on time from enrolment to diagnosis. Both studies are using the same electronic clinical record forms developed by MedSciNet and thus will have a large cross-over of data. The advantage of having these two similar RCTs conducted almost simultaneously is that robust information on the impact of incorporation of PlGF into clinical care will be generated. In addition, the potential exists for a collaborative project such as an individual participant data meta-analyses in the future.

\section{DECLARATIONS}

\section{Ethics approval and consent to participate}

The trial is being conducted in accordance with ethical principles that have their origin in the Declaration of Helsinki and are consistent with Good Clinical Practice (GCP) and applicable regulatory requirements. The local ethics committee at each participating site has reviewed the trial protocol, including the patient information and informed consent form, and full ethical approval granted. Each eligible woman identified is required to give written informed consent prior to her inclusion in the trial. A GCP trained researcher at the local site obtains this consent.

Clinical Research Ethics Committee Cork: ECM 3 (h) 08/11/16.

University College Hospital Galway EC: Ref 50/12.

Coombe Womens \& Infants University Hospital EC: Study No 20-2016.

National Maternity Hospital EC: EC 20.2016.

University Hospital Limerick EC: Ref: 68/16.

Health Research Authority (Belfast): 16/WM/0484.

Rotunda Hospital EC: REC-2016-020.

\section{Dissemination}

The success of the trial will be dependent entirely on the collaboration of clinicians in the participating hospitals and those who hold key responsibility for the trial. Hence, the credit for the study will be assigned to the key collaborator(s) from a participating site as it is crucial that those taking credit for the work have actually carried it out. The results of the trial will be reported first to trial collaborators. The results from the PARROT Ireland trial will be published in an established peer-reviewed journal. At least one publication of the main results will be made. Links to the publication will be provided in all applicable trial registers. Dissemination of results to participants will take place via the media, trial website and relevant participant organisations. Collaborating investigators will play a vital role in disseminating the results to colleagues and participants.

\section{Author affiliations}

${ }^{1}$ The Irish Centre for Fetal and Neonatal Translational Research (INFANT), UCC, Cork, Ireland

${ }^{2}$ University College Cork, Department of Obstetrics and Gynaecology, Cork, Ireland

${ }^{3}$ Public Health, University of Birmingham, Birmingham, UK

${ }^{4}$ Rotunda Hospital, Royal College of Surgeons in Ireland, Dublin, Ireland 
${ }^{5}$ Department of Obstetrics and Gynaecology, Graduate Entry Medical School, University of Limerick, Limerick, Ireland

${ }^{6}$ HRB Trials Methodology Research Network, Dublin, Ireland

${ }^{7}$ National University of Ireland, Galway, Galway, Ireland

${ }^{8}$ Royal Maternity Hospital, Belfast, UK

${ }^{9}$ UCD Perinatal Research Centre, School of Medicine, University College Dublin, National Maternity Hospital, Dublin, Ireland

${ }^{10}$ Obstetrics and Gynaecology, National University of Ireland Galway, Galway, Ireland

${ }^{11}$ Trinity College Dublin \& Coombe Women \& Infants University Hospital Dublin 8,

Republic of Ireland, Dublin, Ireland

${ }^{12}$ Department of Epidemiology and Public Health, University College Cork, Cork, Ireland

${ }^{13}$ The Irish Centre for Fetal and Neonatal Translational Research (INFANT), University College Cork, Cork, Ireland

${ }^{14}$ Economics Department, University College Cork, Cork, Ireland

${ }^{15}$ Department of Paediatrics and Child Health, University College Cork, Cork, Ireland

${ }^{16}$ Department of Women's and Children's Health, University of Liverpool School of

Life Sciences, Liverpool, UK

Acknowledgements The authors would like to thank PARROT Ireland participants and the associated maternity units and staff for their support and involvement in this study.

Contributors All authors contributed to the overall study design and specific methodologies. LK conceived and designed the study with DD. LK and DHR produced the detailed protocol, with input from all authors. DHR drafted the manuscript with assistance from KH, KOD and LK. All authors have critically read, contributed with inputs and revisions and approved the final manuscript.

Funding The PARROT Ireland trial is funded by the Health Research Board Mother and Baby Clinical Trial Network Ireland (HRB CTN-2014-010). The trial is sponsored by University College Cork, Ireland. Neither the funders nor trial sponsor had a role in the design of the study and will not have any role in analyses, interpretation of the data or decision to submit results.

Competing interests None declared.

Patient consent for publication Not required.

Ethics approval Ethical approval has been granted from each of the seven maternity hospitals involved in the trial. The results of the trial will be presented both nationally and internationally at conference and published in an internationa peer-reviewed journal.

Provenance and peer review Not commissioned; externally peer reviewed.

Open access This is an open access article distributed in accordance with the Creative Commons Attribution Non Commercial (CC BY-NC 4.0) license, which permits others to distribute, remix, adapt, build upon this work non-commercially, and license their derivative works on different terms, provided the original work is properly cited, appropriate credit is given, any changes made indicated, and the use is non-commercial. See: http://creativecommons.org/licenses/by-nc/4.0/.

\section{REFERENCES}

1. Sibai B, Dekker G, Kupferminc M. Pre-eclampsia. The Lancet 2005;365:785-99.

2. Meyer NL, Mercer BM, Friedman SA, et al. Urinary dipstick protein: a poor predictor of absent or severe proteinuria. Am J Obstet Gynecol 1994:170(1 Pt 1):137-41.

3. Waugh JJ, Bell SC, Kilby MD, et al. Optimal bedside urinalysis for the detection of proteinuria in hypertensive pregnancy: a study of diagnostic accuracy. BJOG 2005;112:412-7.

4. Kuo VS, Koumantakis G, Gallery ED. Proteinuria and its assessment in normal and hypertensive pregnancy. Am J Obstet Gynecol 1992;167:723-8.

5. Villar J, Repke J, Markush L, et al. The measuring of blood pressure during pregnancy. Am J Obstet Gynecol 1989;161:1019-24.

6. ACOG practice bulletin. Diagnosis and management of preeclampsia and eclampsia. Obstetrics and gynecology 2002;99:159-67.

7. Meads CA, Cnossen JS, Meher S, et al. Methods of prediction and prevention of pre-eclampsia: systematic reviews of accuracy and effectiveness literature with economic modelling. Health Technol Assess 2008;12:1.

8. Maglione D, Guerriero V, Viglietto G, et al. Isolation of a human placenta cDNA coding for a protein related to the vascular permeability factor. Proc Natl Acad Sci U S A 1991;88:9267-71.
9. de Vries C, Escobedo JA, Ueno $\mathrm{H}$, et al. The fms-like tyrosine kinase, a receptor for vascular endothelial growth factor. Science 1992;255:989-91.

10. Kanno S, Oda N, Abe M, et al. Roles of two VEGF receptors, Flt-1 and KDR, in the signal transduction of VEGF effects in human vascular endothelial cells. Oncogene 2000;19:2138-46.

11. Hayman R, Brockelsby J, Kenny L, et al. Preeclampsia: the endothelium, circulating factor(s) and vascular endothelial growth factor. J Soc Gynecol Investig 1999;6:3-10.

12. Levine RJ, Maynard SE, Qian C, et al. Circulating angiogenic factors and the risk of preeclampsia. N Engl J Med 2004;350:672-83.

13. Chappell LC, Duckworth S, Seed PT, et al. Diagnostic accuracy of placental growth factor in women with suspected preeclampsia a prospective multicenter study. Circulation 2013;128:2121-31.

14. Zeisler H, Llurba E, Chantraine F, et al. Predictive Value of the sFlt1:PIGF Ratio in Women with Suspected Preeclampsia. N Engl J Med Overseas Ed 2016;374:13-22.

15. Zeisler H, Llurba E, Chantraine F, et al. Predictive Value of the sFlt1:PIGF Ratio in Women with Suspected Preeclampsia. N Engl J Med 2016;374:13-22.

16. PIGF-based testing to help diagnose suspected pre-eclampsia (Triage PIGF test, Elecsys immunoassay sFIt-1/PIGF ratio, DELFIA Xpress PIGF 1-2-3 test, and BRAHMS sFIt-1 Kryptor/BRAHMS PIGF plus Kryptor PE ra. Diagnostics guidance [DG23]. 2016 https://www. nice.org.uk/guidance/dg23/chapter/1-Recommendations (Cited May 2016).

17. Hemming K, Haines TP, Chilton PJ, et al. The stepped wedge cluster randomised trial: rationale, design, analysis, and reporting. BMJ 2015;350:h391.

18. Bland JM. Cluster randomised trials in the medical literature: two bibliometric surveys. BMC Med Res Methodol 2004;4:21.

19. Brown CA, Lilford RJ. The stepped wedge trial design: a systematic review. BMC Med Res Methodol 2006;6:54.

20. Stolberg HO, Norman G, Trop I. Randomized controlled trials. AJR Am J Roentgenol 2004;183:1539-44.

21. GCP. ICH Harmonised tripartite guideline, guideline for good clinical practice E6(R1). 1996 https://www.ich.org/fileadmin/Public Web_Site/ICH_Products/Guidelines/Efficacy/E6/E6_R1_Guideline. pdf.

22. HSE. The manangement of hypertension in pregnancy. 2016:1-32.

23. NICE. Hypertension in Pregnancy. 2010 https://www.nice.org.uk/ guidance/cg107.

24. Cantwell R, Clutton-Brock T, Cooper G, et al. Saving Mothers' Lives: reviewing maternal deaths to make motherhood safer: 2006-2008. The Eighth Report of the Confidential Enquiries into Maternal Deaths in the United Kingdom. BJOG 2011;118(Suppl 1):1-203.

25. Schutte JM, Schuitemaker NW, van Roosmalen J, et al. Dutch Maternal Mortality Committee. Substandard care in maternal mortality due to hypertensive disease in pregnancy in the Netherlands. BJOG 2008;115:732-6.

26. Martin JN, Thigpen BD, Moore RC, et al. Stroke and severe preeclampsia and eclampsia: a paradigm shift focusing on systolic blood pressure. Obstet Gynecol 2005;105:246-54.

27. Sharp A, Chappell L, Dekker G, et al. OP 2 Placental growth factor informed management of suspected pre-eclampsia and/or fetal growth restriction - The MAPPLE cohort study. Pregnancy Hypertens 2017:9:9-10.

28. EQ-5D. www.euroqol.org.

29. Measuring and Valuing Health. SF 6D. https://www.sheffield.ac.uk/ scharr/sections/heds/mvh/sf-6d.

30. EuroQol Group. EuroQol-a new facility for the measurement of health-related quality of life. Health Policy 1990;16:199-208.

31. Ware JE, Sherbourne CD. The MOS 36-item short-form health survey (SF-36). I. Conceptual framework and item selection. Med Care 1992;30:473-83.

32. Brazier J, Usherwood T, Harper R, et al. Deriving a preference-based single index from the UK SF-36 Health Survey. J Clin Epidemiol 1998;51:1115-28.

33. Chappell LC, Duckworth S, Seed PT, et al. Diagnostic accuracy of placental growth factor in women with suspected preeclampsia: a prospective multicenter study. Circulation 2013;128:2121-31.

34. Martin J, Girling A, Nirantharakumar K, et al. Intra-cluster and interperiod correlation coefficients for cross-sectional cluster randomised controlled trials for type-2 diabetes in UK primary care. Trials 2016;17:402.

35. Hooper R, Bourke L. Cluster randomised trials with repeated cross sections: alternatives to parallel group designs. BMJ 2015;350:h2925.

36. Hemming K. The Shiny CRT Calculator: power and sample size for cluster randomised trials. https://clusterrcts.shinyapps.io/rshinyapp/. 
37. Campbell MK, Fayers PM, Grimshaw JM. Determinants of the intracluster correlation coefficient in cluster randomized trials: the case of implementation research. Clin Trials 2005;2:99-107.

38. Hemming K, Taljaard M. Sample size calculations for stepped wedge and cluster randomised trials: a unified approach. $J$ Clin Epidemiol 2016;69:137-46.

39. Hemming K, Eldridge S, Forbes $\mathrm{G}$, et al. How to design efficient cluster randomised trials. BMJ 2017;358:j3064.
40. Hemming K, Taljaard M, Forbes A. Analysis of cluster randomised stepped wedge trials with repeated cross-sectional samples. Trials 2017; 18:101.

41. Zou G. A modified poisson regression approach to prospective studies with binary data. Am J Epidemiol 2004;159:702-6.

42. Duley L. The global impact of pre-eclampsia and eclampsia. Semin Perinatol 2009;33:130-7. 